\author{
EUROPEAN ORGANIZATION FOR NUCLEAR RESEARCH \\ European Laboratory for Particle Physics
}

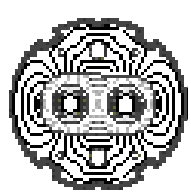

Large Hadron Collider Project

LHC Project Report 650

\title{
WAVEGUIDE REFLECTOMETRY FOR OBSTACLE DETECTION IN THE LHC BEAM PIPE INCLUDING SIGNAL ATTENUATION
}

\author{
F. Caspers, T. Kroyer, A.Mostacci
}

\begin{abstract}
Unexpected obstacle formation in the LHC beam-pipe during assembly, cool down and operation may lead to major disturbances. Thus a fast, precise, sensitive and reliable means to detect and characterize such a fault is highly desirable, preferably without the need to break the vacuum. Waveguide mode time domain reflectometry using the synthetic pulse technique has been selected for this purpose. The system will use a modern vector network analyzer operating using essentially the fundamental TM mode on the LHC beam-screen. The objective is to measure over a full arc with access from either side both in reflection and transmission mode. If the proposed system is implemented a total of 32 couplers will be permanently installed, which may be used in normal operation for beam diagnostics and other applications.

The attenuation of several short beam-screen sections has been measured both for TE and TM modes by means of a resonator method and these data are compared with theoretical results. Waveguide calibration on the beam-pipe and digital signal processing to compensate dispersion are studied. Finally, using a $44 \mathrm{~m}$ test track the performance of the proposed methods is examined.
\end{abstract}

$\begin{array}{ll}\text { Presented at } & \text { PAC 2003, } \\ & \text { Portland, Oregon, USA, } \\ & \text { from } 12 \text { to } 16 \text { May 2003 }\end{array}$

CERN

CH - 1211 Geneva 23

Switzerland

Geneva, 


\title{
WAVEGUIDE MODE REFLECTOMETRY FOR OBSTACLE DETECTION IN THE LHC BEAM PIPE INCLUDING SIGNAL ATTENUATION
}

\author{
F. Caspers, T. Kroyer, CERN, Geneva, Switzerland
}

\begin{abstract}
Unexpected obstacle formation in the LHC beam-pipe during assembly, cool down and operation may lead to major disturbances. Thus a fast, precise, sensitive and reliable means to detect and characterize such a fault is highly desirable, preferably without the need to break the vacuum. Waveguide mode time domain reflectometry using the synthetic pulse technique has been selected for this purpose. The system will use a modern vector network analyzer operating using essentially the fundamental TM mode on the LHC beam-screen. The objective is to measure over a full $\operatorname{arc}(2.5 \mathrm{~km})$ with access from either side both in reflection $(\approx 1.25 \mathrm{~km}$ range $)$ and transmission mode. If the proposed system is implemented a total of 32 couplers will be permanently installed, which may be used in normal operation for beam diagnostics and other applications.

The attenuation of several short beam-screen sections has been measured both for TE and TM modes by means of a resonator method and these data are compared with theoretical results. Waveguide calibration on the beam-pipe and digital signal processing to compensate dispersion are studied. Finally, using a $44 \mathrm{~m}$ test track the performance of the proposed methods is examined.
\end{abstract}

\section{INTRODUCTION}

For proper operation of CERN's Large Hadron Collider, it is of utmost importance that the beam-screen, fig. 1, is free from any obstacle larger than a pinhead. During the installation procedure however, there is a non-negligible probability that a permanent deformation or simply some forgotten screw remains inside. In this case it would be advantageous to have a means to detect such obstacles during assembly and possibly in situ without having to open the vacuum.

Any intruding device such as a little robot is out of question for vacuum reasons, regardless of the possibility that it could get stuck inside. Optical lasers and ultrasonic pulse-echo detection are too limited in dynamic range. However the beam-pipe can be considered as a waveguide with modes propagating above a certain cutoff frequency. Since the beam-pipe is covered with axial pumping slots, TE modes will radiate considerably, while TM modes are hardly influenced, given that their wall currents are parallel to the slots.

In this approach synthetic pulse reflectometry was preferred over a $\mathrm{FMCW}^{1}$ method and real time pulse reflectometry for the simple reason that the latter methods would require the development of much of the front-end measure-

\footnotetext{
${ }^{1}$ Frequency Modulated Continuous Wave
}

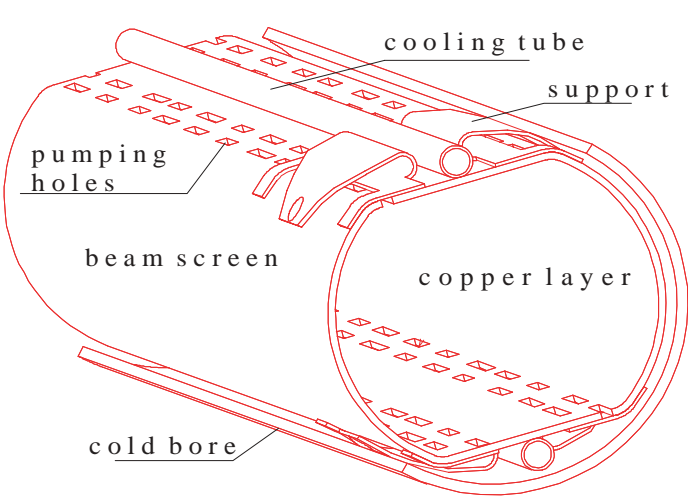

Figure 1: The LHC beam-screen with the surrounding cold bore, the pumping slots and the cooling tubes

ment equipment. For synthetic pulse reflectometry a stateof-the-art vector network analyser (VNA) has been used to measure the frequency response of $s_{11}$, the time domain profile of $s_{11}$ is obtained by Fourier transform. In addition, high dynamic range $(>120 \mathrm{~dB})$ and good reproducibility have been obtained this way. This method has already been successfully applied over much shorter ranges $(13 \mathrm{~m})$ at $\operatorname{ESRF}^{2}[1]$.

Two plans for the realization are proposed:

- The baseline programme: During assembly, sections of say $100 \mathrm{~m}$ length are checked before sealing the beam-pipe. The measurement would be done at room temperature by branching some kind of adaptor directly on one end of the beam-pipe.

- The full scale version should allow in-situ measurements in the LHC. Coupling to the beam-pipe should utilize a button type pick-up installed at the end of each arc (32 in total), requiring detection ranges up to $1250 \mathrm{~m}$ at low temperature.

\section{RESONATOR MEASUREMENTS}

The attenuation in the beam-pipe constitutes a major limiting factor. We used a resonator method to determine the attenuation and the cut-off frequencies of the first TE and TM modes. The resonator was built by soldering brass plates on the ends of a sample of beam-pipe. A measurement in transmission mode reveals the characteristic pattern of peaks as shown in fig. 2 .

\section{Cut-off frequencies}

Given this data, the cut-off frequency of the respective mode can be determined with very good accuracy using

\footnotetext{
${ }^{2}$ European Synchrotron Radiation Facility, based at Grenoble, France
} 


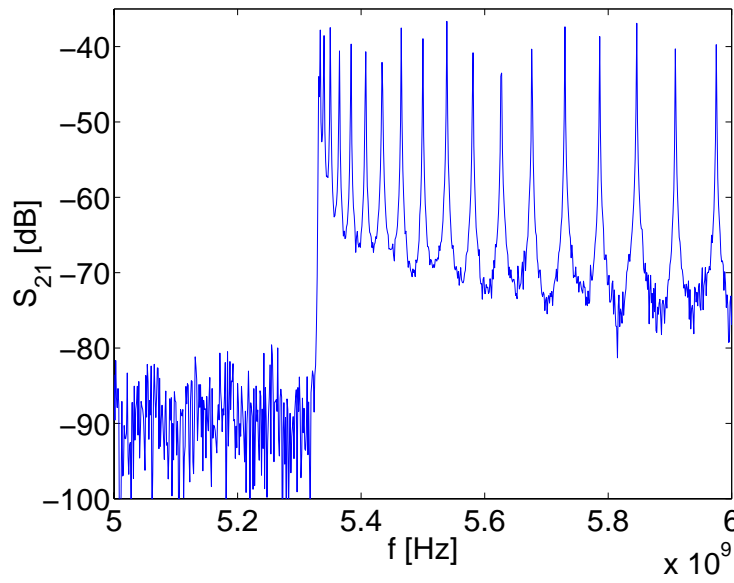

Figure 2: Pattern of resonance peaks for the first TM mode on a $100 \mathrm{~cm}$ long beam-pipe resonator in transmission

the method presented in [2]. The cut-off of the first TE and TM modes are given in the table below.

\begin{tabular}{c||c|c|c|c} 
Mode $^{3}$ & $T E_{c 11}$ & $T E_{s 11}$ & $T M_{01}$ & $T M_{11}$ \\
\hline$f_{c}[\mathrm{GHz}]$ & 3.61 & 4.32 & 5.33 & 7.99
\end{tabular}

Table 1: The measured cut-off frequencies for the first modes on the slotted beam-pipe

\section{Attenuation}

The relation between a peak's quality factor and the attenuation at this frequency is given in [3] by

$$
Q_{L}=\frac{\pi \lambda_{H}}{\lambda_{0}^{2} \alpha}
$$

with $Q_{L}, \lambda_{H}, \lambda_{0}$ and $\alpha$ representing the measured loaded quality factor, the guided wavelength, the free wavelength and the attenuation in $[\mathrm{dB} / \mathrm{m}]$, respectively. By appropriate choice of the mode launchers the coupling was kept sufficiently small that the unloaded quality factor $Q_{0}$ could be approximated by $Q_{L}$.

In figure 3 the attenuation $\alpha$ for the fundamental TM mode is compared to the theoretical curve for a circular waveguide having the same cut-off frequency $f_{c}$, as given in [4]. The measured values are larger by around $40 \%$, which could be due to the properties of the beam-pipe, especially the longitudinal welding strip, the saw-teeth like corrugations, the general surface roughness and to a minor degree, the radiation through the slots.

For the TE modes ${ }^{3}$ things look different. The $T E_{s 11}$ mode is much affected by the slots, since its current maximum is at the location of the slots. The radiation losses make the attenuation increase by nearly a factor 2 . For the fundamental TE mode $\left(T E_{c 11}\right)$ this is not the case, however its attenuation curve flattens above $4.5 \mathrm{GHz}$, which indicates

\footnotetext{
${ }^{3}$ The indices $c$ and $s$ of the stand for the sine (vertical) and cosine (horizontal) polarization of the $T E_{11}$ mode
}

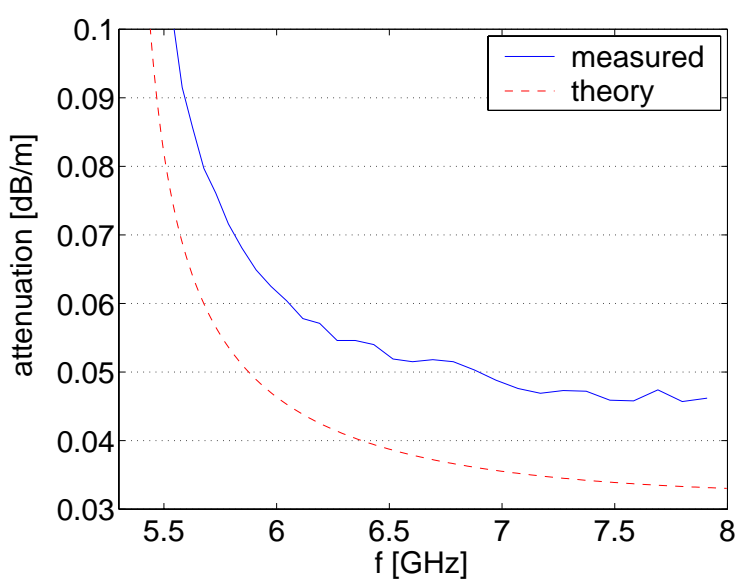

Figure 3: Attenuation of the fundamental TM mode on the beam-pipe, compared to calculated values for a circumscribed circular waveguide

that radiation losses (which increase with $f^{2}$ as a first approximation) begin to prevail. At low temperature (20K) this effect will become more significant, since the resistive losses are estimated to decrease by a factor 4 to 5 . This observation indicates that the $T M_{01}$ mode should be a better choice than the fundamental TE mode.

\section{MEASUREMENTS ON THE TEST TRACK}

After some preliminary tests a straight section of nearly $44 \mathrm{~m}$ of beam-pipe with two interconnects was made available for measurements. The coupling to the beam-pipe consisted of a non-optimized adapter available from previous tests that was fitted to the end of the beam-pipe. Inserting an appropriate mode launcher excited the desired mode. On this line the performance of waveguide calibration and signal processing was tested.

\section{Waveguide calibration}

When doing measurements involving transitions between different types of lines and on electrically long devices calibration is highly recommended. For such a nonstandard waveguide profile as the beam-pipe however no calibration standards are commercially available. Therefore we had to make them on our own. As for an ordinary waveguide calibration, three standards were used: two offset shorts of lengths $l_{1} \approx 7 \mathrm{~mm}$ and $l_{2} \approx 20 \mathrm{~mm}$ and one load. The shorts were realized by soldering a brass plate at the given position into a sample of beam-pipe and to get a load, some absorbing material was inserted into another piece (fig. 4).

The major advantage of waveguide calibration on the beam-pipe was found to be that initial reflections from the beam-pipe adapter could be avoided. However, due to the fragile contacts of the adapter and changing properties of cables, the calibration deteriorated every time some mechanical intervention was done. In particular in the time 


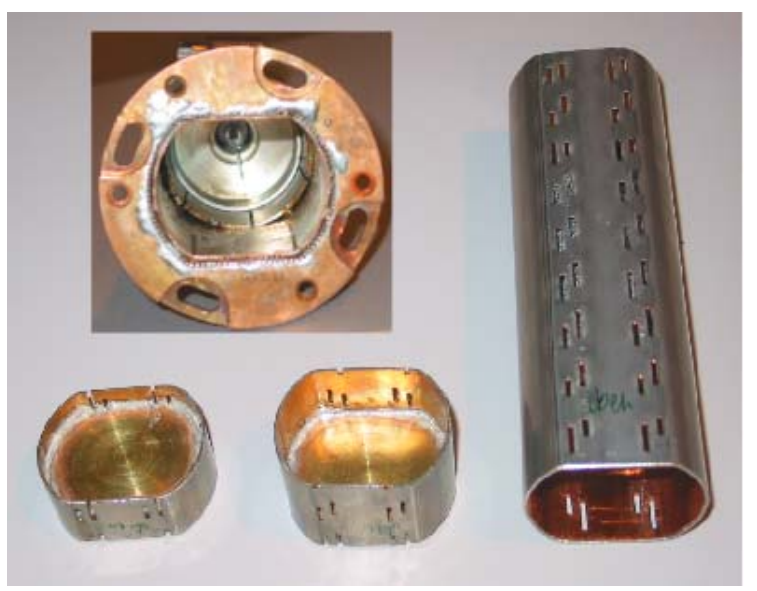

Figure 4: The coax-to-beam-pipe adapter and the three standards for waveguide calibration

domain it was noticed that calibrated measurements have a smaller dynamic range ${ }^{4}$ than uncalibrated ones.

\section{Signal processing}

It soon became clear that some serious signal processing had to be done. This is mainly due to two reasons:

- The maximum number of points available on our VNA is limited to 1601 . Since this is insufficient to get both good spatial resolution (large frequency range) and good spatial range (closely spaced frequency points), measurements in adjacent frequency ranges have to be combined externally before doing the FFT.

- Waveguide dispersion on the beam-pipe will cause considerable smear-out on the emitted (synthetic) pulses. A numerical correction of this effect is called for.

For the implementation of these tasks MATLAB C was chosen.

Dispersion compensation. After having run down $z$ meters on a waveguide with guided wavelength $\lambda_{H}$ and cut-off frequency $f_{c}$ the phase change can be expressed by

$\phi(f)=-\frac{2 \pi}{\lambda_{H}} z=-\frac{2 \pi f \sqrt{1-\left(\frac{f_{c}}{f}\right)^{2}}}{c_{0}} z=-2 \pi \tau \sqrt{f^{2}-f_{c}^{2}}$,

introducing the free space delay $\tau=\frac{z}{c_{0}}$. By subtracting the part of this phase term that goes with higher than linear order of $f$ from the phase of measured data, the dispersion can be compensated for one distance $z$. By repeatedly doing this procedure for increasing foci $z$, each time cutting out the focused region a dispersion compensated trace can be made up. The actual performance of this method is illustrated in figure 5 .

\footnotetext{
${ }^{4}$ difference between maximum amplitude and RMS noise level in one time domain trace
}

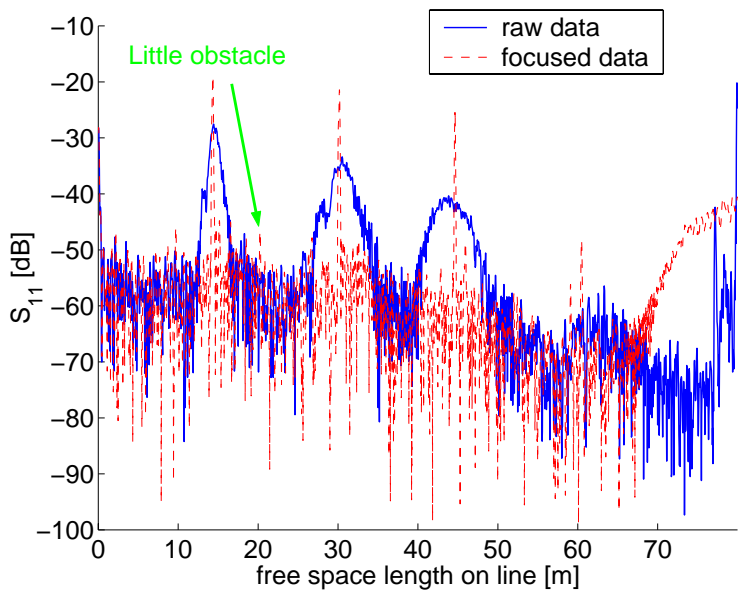

Figure 5: Dispersion compensation for the first TM mode on the test track

Other implemented features, such as analysis of mode mixing and multiple reflections by the interconnects are presented in detail in [5].

\section{CONCLUSION}

The feasibility of an obstacle-locating reflectometer for the LHC beam-pipe was studied. First the crucial characteristics of the beam-pipe were determined in a resonator measurement. With the attenuation obtained, a range of $600 \mathrm{~m}$ for the detection of a M4 nut size obstacle seems possible at room temperature, with a substantial gain expected for low temperature operation (20K). In a measurement on a $44 \mathrm{~m}$ long test track, waveguide calibration on the beampipe was examined and the data was processed to correct smear-out provoked by dispersion.

\section{ACKNOWLEDGEMENTS}

The authors would like to thank F. Peterson, T. Linnecar and R. Garoby for their support and N. Hilleret, J. Knaster, E. and H. Kos for their help.

\section{REFERENCES}

[1] Caspers, F., Jacob, J., Gautier, G. et alias, Wireless Impedance Measurements and Fault Location on ESRF Vacuum Chamber Assemblies, CERN/PS, 1992

[2] Caspers, Fritz and Scholz, T., Measurement of Trapped Modes in Perforated Waveguides, CERN-PS-RF, 2000

[3] Matthaei, George, Young, Leo and Jones, E. M. T., Microwave Filters, Impedance-Matching Networks, and Coupling Structures, Artech House, Dedham, 1980

[4] Meinke, H. and Gundlach, F. W., Taschenbuch der Hochfrequenztechnik, Dritte Auflage, Springer-Verlag, Berlin, 1968

[5] Kroyer, Thomas, A Waveguide High Order Mode Reflectometer for the Large Hadron Collider Beam-pipe, Wien, 2003 (to be published) 